PROCEEDINGS OF THE

AMERICAN MATHEMATICAL SOCIETY

Volume 135, Number 9, September 2007, Pages 2961-2965

S 0002-9939(07)08932-0

Article electronically published on May 10, 2007

\title{
THE CONFORMAL DEFORMATION DETOUR COMPLEX FOR THE OBSTRUCTION TENSOR
}

\author{
THOMAS P. BRANSON AND A. ROD GOVER
}

(Communicated by Jon G. Wolfson)

The second author dedicates the paper to the memory of Thomas P. Branson (1953-2006)

\begin{abstract}
On pseudo-Riemannian manifolds of even dimension $n \geq 4$, with everywhere vanishing (Fefferman-Graham) obstruction tensor, we construct a complex of conformally invariant differential operators. The complex controls the infinitesimal deformations of obstruction-flat structures, and, in the case of Riemannian signature the complex is elliptic.
\end{abstract}

\section{INTRODUCTION}

Ever since its introduction, as the natural tensor controlling a conformal relativity [1], the Bach tensor has been considered an intriguing and important natural invariant in 4-dimensional Riemannian and pseudo-Riemannian geometry. In higher even dimensions $n$, an analogue of the Bach tensor was discovered by Fefferman and Graham [12]; it arose as an obstruction to their ambient metric construction. This Fefferman-Graham obstruction tensor, which we denote $\mathcal{B}_{a b}$, is a natural tensor that shares many of the properties of the Bach tensor. It is a conformally invariant, trace-free and divergence-free symmetric 2-tensor that vanishes for conformally Einstein metrics. Recently Graham and Hirachi [15] have shown that, also generalising the situation in dimension $4, \mathcal{B}_{a b}$ is the total metric variation of $\int Q$, where $Q$ is Branson's Q-curvature 3. Along the way they develop many of the properties of $\mathcal{B}_{a b}$. These properties are also developed in [15] via an alternative, but equivalent, definition of the obstruction; it is given as the image of a certain conformal operator when applied to the Weyl curvature.

On conformally flat manifolds a large class of conformally invariant operators between irreducible bundles are organised into differential complexes known as generalised Bernstein-Gelfand-Gelfand (gBGG) complexes. Recently there has been spectacular progress in the construction of curved analogues of these and analogous operators for other parabolic geometries; see [7, 9] and references therein. The issue of when these or other invariant operators may be combined to yield a complex, in some curved setting, is more subtle, and there are only limited results. On

Received by the editors June 13, 2006.

2000 Mathematics Subject Classification. Primary 53A30; Secondary 53A55.

Key words and phrases. conformal differential geometry, elliptic operators.

Both authors would like to thank the Mathematical Sciences Research Institute, Berkeley.

The second author would also like to thank the Royal Society of New Zealand for support via Marsden Grant no. 02-UOA-108.

(C)2007 American Mathematical Society 
half-flat conformal 4-manifolds there is a well-known systematic construction of a class of complexes. This has been extended to other structures by various authors and, recently, a rather general theory produced by Čap and Souček [8]. Some time ago it was observed by the current authors that, from the classification of conformally flat operators on the sphere 2, 11, it follows easily that on conformally flat structures there is a huge class of complexes related to gBGG complexes. We term these detour complexes, and each such is closely related to a gBGG complex. In [5] we show that for the de Rham complex the corresponding detours yield conformally invariant differential complexes even in the fully curved case. These complexes are related to a conformal Hodge theory and yield new global invariants 6. In [14, as a background to the treatment of the obstruction tensor, some examples of other detour complexes are discussed in the conformally flat setting. These are related to deformations of conformally flat structures. It is in general a severe condition to require that a sequence of differential operators both be a complex, and conformally invariant; in general we do not expect complexes from conformally flat structures to admit curved analogues. Nevertheless we show that one of the complexes from 14. may be extended to curved structures that satisfy the curvature condition of the everywhere vanishing Fefferman-Graham obstruction tensor. Background for much of the discussion here may be found in 4. Discussions with Andrew Waldron on related issues have been much appreciated.

\section{The DeFormation Detour COMPLEX FOR THE OBSTRUCTION}

We work on a pseudo-Riemannian manifold $M$ of even dimension $n \geq 4$. The metric will be denoted by $g$. All bundles, section spaces and structures will be smooth. Let us write $T$ and $T^{*}$ for, respectively, the tangent bundle and the cotangent bundle (although we will also identify these via the metric without further comment). Then $S_{0}^{2} T^{*}$ denotes the trace-free part of the second symmetric tensor power of $T^{*}$. We will use the same notation for section spaces.

On a tangent vector field $X$, the Killing operator is the first order differential operator $X \mapsto \mathcal{L}_{X} g$, where $\mathcal{L}_{X}$ denotes the Lie derivative along the flow of $X$. We write $K X$ as a shorthand for this Killing operator on $X$, and, taking traces via the metric and its inverse, we write $K_{0} X$ for the trace-free part of $K X$. The operator $X \mapsto K_{0} X$ is known as the conformal Killing operator, and it is well-known that this is conformally invariant. We write $K_{0}^{*}$ for the formal adjoint of this operator.

Let us write $B$ (or sometimes $B^{g}$ ) for the linearisation, at the metric $g$, of the operator which takes metrics $g$ to $\mathcal{B}^{g}$. Since $\mathcal{B}$ is trace-free symmetric it follows that $B$ also takes values in $S_{0}^{2} T^{*}$ on manifolds with $\mathcal{B}=0$ everywhere. The main result is the following.

Theorem 2.1. On pseudo-Riemannian manifolds with the Fefferman-Graham obstruction tensor vanishing everywhere, the sequence of operators

$$
T \stackrel{K_{0}}{\rightarrow} S_{0}^{2} T^{*} \stackrel{B}{\rightarrow} S_{0}^{2} T^{*} \stackrel{K_{0}^{*}}{\rightarrow} T
$$

is a formally self-adjoint complex of conformally invariant operators. In Riemannian signature the complex is elliptic. 
Proof. Since the conformal Killing operator $K_{0}$ is conformally invariant, so is its formal adjoint $K_{0}^{*}$. Viewing $\mathcal{B}$ as a tensor for each metric (rather than a densityvalued tensor), we have

$$
\mathcal{B}^{\widehat{g}}=e^{(2-n) \Upsilon} \mathcal{B}^{g}
$$

where $\widehat{g}=e^{2 \Upsilon} g$ for a smooth function $\Upsilon$. Thus setting $\Upsilon=t \omega$, for a real parameter $t$, and differentiating with respect to $t$ at $t=0$, we obtain the result that,

$$
B(\omega g)=\frac{2-n}{2} \omega \mathcal{B}^{g}
$$

for any function $\omega$. Thus if $\mathcal{B}=0$, then $B(\omega g)=0$; since $B$ annihilates pure trace terms it descends to an operator $S_{0}^{2} T^{*} \rightarrow S_{0}^{2} T^{*}$. From (2), $B^{g}$ is conformally bi-invariant as follows:

$$
B^{\widehat{g}}\left(e^{2 \Upsilon} h\right)=e^{(2-n) \Upsilon} B^{g} h,
$$

for $h$ a section in $S_{0}^{2} T^{*}$. So the sequence is conformally invariant as claimed.

Since the obstruction tensor $\mathcal{B}^{g}$ is a natural Riemannian invariant, the Lie derivative along a vector field $X$ is

$$
\mathcal{L}_{X} \mathcal{B}=B\left(\mathcal{L}_{X} g\right)=B(K X),
$$

by the chain rule. We have seen that, under the curvature condition $\mathcal{B}=0, B$ annihilates terms that are pure trace and so we have $B(K X)=B\left(K_{0} X\right)=0$. That is, the composition $B K_{0}$ is zero.

As mentioned above, the obstruction tensor $\mathcal{B}$ arises as the total metric variation of the integral of $Q$. Thus its linearisation is a second variation of $\int Q$ and hence is a formally self-adjoint operator on the space of metric variations. So $B$ is formally self-adjoint on $S_{0}^{2} T^{*}$. Thus the sequence (1) is formally self-adjoint and hence a complex.

We write $\nabla$ for the Levi-Civita connection, and $\Delta$ for the Laplacian $g^{a b} \nabla_{a} \nabla_{b}$ (given here in abstract index notation). Note that from [15, 14] we have

$$
\mathcal{B}=\Delta^{n / 2-2} \delta^{\nabla} \delta^{\nabla} C+\mathrm{LOT},
$$

where $C$ is the Weyl curvature tensor, $\delta^{\nabla}$ indicates the obvious covariant divergence operators (in an abstract index notation $\delta^{\nabla} \delta^{\nabla} C$ is $\nabla^{b} \nabla^{d} C_{a b c d}$ ), and LOT indicates lower order terms. Thus at leading order its linearisation takes the form

$$
B=\Delta^{n / 2-2} \delta^{\nabla} \delta^{\nabla} W+\mathrm{LOT},
$$

where $W$ is the linearisation of the Weyl curvature, as a differential operator on $S_{0}^{2} T^{*}$. But from elementary classical invariant theory it is easily verified that the double divergence is the formal adjoint of the operator $W$. This may be commuted past the Laplacian power at the expense of lower order terms and so the leading term of $B$ is simply

$$
W^{*} \Delta^{n / 2-2} W
$$

and whence, in the case of Riemannian signature, the leading symbol of $B, \sigma(B)$ has the same kernel as $\sigma(W)$. This kernel is the same as the image space of the leading symbol of $K_{0}$ since these give the leading terms of a resolution [13, 9, 14]

$$
T \stackrel{K_{0}}{\rightarrow} S_{0}^{2} T^{*} \stackrel{W}{\rightarrow} \mathcal{W} \rightarrow \cdots
$$


that controls deformations of conformally flat structures. Here $\mathcal{W}$ is the space of algebraic Weyl tensors. Thus the symbol sequence is exact and the complex (1) is elliptic as claimed.

Remarks. Since the operator $K_{0}$ takes infinitesimal diffeomorphisms to their action on conformal structure, it is clear that the first cohomology of the complex (11) is the formal tangent space to the moduli space of deformations of obstruction-flat geometries. In fact the complex controls the full formal deformation theory, but this will be taken up elsewhere.

To simplify the exposition here we avoided the use of conformal densities and discussion of conformal structures. Writing $E[w]$ for the bundle of conformal densities of weight $w$ (see e.g. [10]), and $T^{*}[w]$ as a shorthand for $T^{*} \otimes E[w]$ and so forth, the complex is

$$
T \stackrel{K_{0}}{\rightarrow} S_{0}^{2} T^{*}[2] \stackrel{B}{\rightarrow} S_{0}^{2} T^{*}[2-n] \stackrel{K_{0}^{*}}{\rightarrow} T^{*}[-n]
$$

or, in the notation of [14] (where the conformally flat version of this is discussed),

$$
\mathcal{E}^{1}[2] \stackrel{K_{0}}{\rightarrow} \mathcal{E}^{1,1}[2] \stackrel{B}{\rightarrow} \mathcal{E}_{1,1}[-2] \stackrel{K_{0}^{*}}{\rightarrow} \mathcal{E}_{1}[-2] .
$$

\section{ACKNOWLEDGMENTS}

The main idea for the dimension 4 version of this complex was developed during the Spring 2001 programme "Spectral Invariants-Analytical and Geometric Aspects" at the Mathematical Sciences Research Institute, Berkeley, California. This support by the MSRI is greatly appreciated.

\section{REFERENCES}

1. R. Bach, Zur Weylschen Relativitätstheorie und der Weylschen Erweiterung des Krümmungstensorbegriffs, Math. Z. 9 (1921), 110-135. MR.1544454

2. B.D. Boe and D.H. Collingwood, A comparison theory for the structure of induced representations, J. Alg. 94 (1985), 511-545. MR0792968 (87b:22026a)

3. T. Branson, Sharp inequalities, the functional determinant, and the complementary series. Trans. Amer. Math. Soc. 347 (1995), 3671-3742. MR.1316845 (96e:58162)

4. T. Branson, Q-curvature and spectral invariants. Rend. Circ. Mat. Palermo (2) Suppl. No. 75 (2005), 11-55. MR2152355 (2006h:53024)

5. T. Branson, and A.R. Gover, Conformally invariant operators, differential forms, cohomology and a generalisation of Q-curvature, Comm. Partial Differential Equations, 30 (2005), 16111669. MR 2182307 (2006k:58060)

6. T. Branson, and A.R. Gover, Pontrjagin forms and invariant objects related to the Qcurvature, Communications in Contemporary Math., to appear.

7. A. Čap, J. Slovák, and V. Souček, Bernstein-Gelfand-Gelfand sequences, Ann. of Math. 154 (2001), 97-113. MR.1847589(2002h:58034)

8. A. Čap, and V. Souček, Subcomplexes in Curved BGG-Sequences. Preprint math.DG/0508534, http://arXiv.org.

9. D.M.J. Calderbank, and T. Diemer, Differential invariants and curved Bernstein-GelfandGelfand sequences J. Reine Angew. Math. 537 (2001), 67-103. MR1856258 (2002k:58048)

10. A. Čap, and A.R. Gover, Standard tractors and the conformal ambient metric construction, Ann. Global Anal. Geom. 24 (2003), 231-259. MR:1996768 (2004g:53016)

11. M.G. Eastwood and J. Slovák, Semiholonomic Verma modules. J. Algebra 197 (1997), 424448. MR:1483772 (98k:22054)

12. C. Fefferman and C.R. Graham, Conformal invariants. The mathematical heritage of Élie Cartan (Lyon, 1984). Astérisque 1985, Numero Hors Série, 95-116. MR0837196 (87g:53060) 
13. J. Gasqui and H. GoldSchmidt, Déformations infinitésimales des structures conformes plates (French) [Infinitesimal deformations of flat conformal structures]. Progress in Mathematics, 52. Birkhäuser Boston, Inc., Boston, MA, 1984. MR0776970 (86m:58156)

14. A.R. Gover and L.J. Peterson, The ambient obstruction tensor and the conformal deformation complex, Pacific J. Math., 226 (2006), 309-351. MR2247867

15. C.R. Graham and K. Hirachi, The ambient obstruction tensor and Q-curvature, pp 59-71. AdS-CFT Correspondence: Einstein Metrics and their Conformal Boundaries. IRMA Lect. Math. Theor. Phys. 8. MR2160867 (2006k:53045)

Department of Mathematics, The University of Iowa, Iowa City, Iowa 52242

Department of Mathematics, The University of Auckland, Private Bag 92019, AuckLAND 1, New Zealand

E-mail address: gover@math.auckland.ac.nz 